\title{
Nucleocapsid-like Structures of Ebola Virus Reconstructed Using Electron Tomography
}

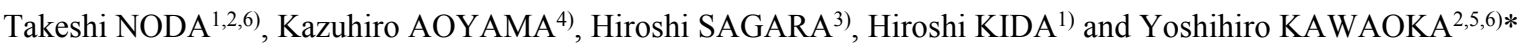 \\ ${ }^{1)}$ Laboratory of Microbiology, Department of Disease Control, Graduate School of Veterinary Medicine, Hokkaido University, Sapporo \\ 060-0818, 2) Division of Virology, Department of Microbiology and Immunology, ${ }^{3)}$ Fine Morphology Laboratory, Department of Basic \\ Medical Science, Institute of Medical Science, The University of Tokyo, Shirokanedai, Minato-ku, Tokyo 108-8639, ${ }^{4}$ Application \\ Laboratory, FEI Company Japan Ltd., Japan, ${ }^{5}$ Department of Pathobiological Science, School of Veterinary Medicine, University of \\ Wisconsin-Madison, Madison, Wisconsin 53706, U.S.A. and ${ }^{6)}$ CREST, Japan Science and Technology Agency, Saitama 332-0012, Japan
}

(Received 1 October 2004/Accepted 5 November 2004)

ABSTRACT. Electron tomography (ET) is a new technique for high resolution, three-dimensional (3D) reconstruction of pleiomorphic macromolecular complexes, such as virus components. By employing this technique, we resolved the 3D structure of Ebola virus nucleocapsid-like (NC-like) structures in the cytoplasm of cells expressing NP, VP24, and VP35: the minimum components required to form these NC-like structures. Reconstruction of these tubular NC-like structures of Ebola virus showed them to be composed of left-handed helices spaced at short intervals, which is structurally consistent with other non-segmented negative-strand RNA viruses.

KEY WORDS: Ebola virus, electron tomography, nucleocapsid.

Transmission electron microscopy (TEM) has been a useful technique for examining fine structures of a variety of objects, its primary limitation being that the information derived is only in 2 dimensions. Electron tomography (ET), however, allows high resolution, three-dimensional (3D) reconstruction of macromolecular complexes, such as cells and organelles. While the principle of ET was outlined more than three decades ago [4], only recently has the value of this technique been demonstrated and its use gained popularity. ET involves collecting a tilt series of images of an object, which are then computationally combined to obtain a $3 \mathrm{D}$ density map $[1,7]$. In addition, ET permits the visualization of pleiomorphic macromolecules, thereby offering a distinct advantage over single particle analysis, which is restricted to the reconstruction of symmetrical objects. Thus, ET is a powerful tool for acquiring $3 \mathrm{D}$ images of virus components and their spatial arrangement within infected cells.

Ebola virus is an enveloped, non-segmented, negativestrand RNA virus. It is a member of the family Filoviridae, in the order of Mononegavirales [13] and causes a syndrome known as hemorrhagic fever. Its enveloped virions are filamentous, measuring approximately $80 \mathrm{~nm}$ in diameter. Along the central axis of each virion resides a tubular nucleocapsid. As infection progresses, a large number of these nucleocapsids are synthesized in the cytoplasm of the virusinfected cells [8]. The nucleocapsid is thought to be a helical structure ( $50 \mathrm{~nm}$ in diameter), with the helices spaced at $5 \mathrm{~nm}$ intervals. It contains an axial channel of $10-15 \mathrm{~nm}$ in diameter surrounded by a high electron-dense layer of 20 $\mathrm{nm}$ in diameter [6]. Recently, conventional TEM demon-

\footnotetext{
* Correspondence to: Kawaoka, Y., Division of Virology, Department of Microbiology and Immunology, Institute of Medical Science, University of Tokyo, Shirokanedai, Minato-ku, Tokyo 108-8639, Japan.
}

strated that three proteins, nucleoprotein (NP), putative minor matrix protein (VP24), and polymerase cofactor (VP35) were necessary and sufficient for the formation of nucleocapsid-like (NC-like) structures in a mammalian expression system [10].

To obtain 3D structural information on these NC-like structures, we used ET to study NC-like structures present in the cytoplasm of cells expressing NP, VP24, and VP35.

First, to confirm the formation of the NC-like structures, we transfected $10^{6}$ human embryonic kidney (293T) cells with $2 \mu \mathrm{g}$ of pCEZ-NP, $2 \mu \mathrm{g}$ of pCEZ-VP24, and $2 \mu \mathrm{g}$ of pCEZ-VP35, which express NP, VP24, and VP35, respectively [14]. At $72 \mathrm{hr}$ post-transfection, $293 \mathrm{~T}$ cells expressing these viral proteins were prefixed with $2.5 \%$ glutaraldehyde in $0.1 \mathrm{M}$ cacodylate buffer $(\mathrm{pH} 7.4)$, and washed with the same buffer. After being post-fixed with $2 \%$ Osmium tetroxide and washed with distilled water, the specimen was dehydrated using an ethanol gradient and sequential propylene oxide exposure. The specimen was then embedded in Epon 812 Resin mixture, and polymerized at $70^{\circ} \mathrm{C}$ for 2 days. Thin sections were stained with uranyl acetate and lead citrate and subsequently examined with a JEM-1200EX electron microscope at $80 \mathrm{kV}$. These sections revealed NC-like structures in the cytoplasm, as previously reported by Huang et al., 2002. The NC-like structures $(50 \mathrm{~nm}$ in diameter) possessed a central axial space (10-15 $\mathrm{nm}$ in diameter) (Fig. 1A). In addition, they were filamentous and showed helical structures spaced at short intervals (Fig. 1B).

We then carried out a 3D structural analysis of the NClike structures in plasmid-transfected cells. Sections (approximately $200 \mathrm{~nm}$ thick) were affixed on both sides with $10 \mathrm{~nm}$-colloidal gold particles, which served as markers for subsequent image alignment. The grid was then placed on a CompStage specimen holder (FEI Company 

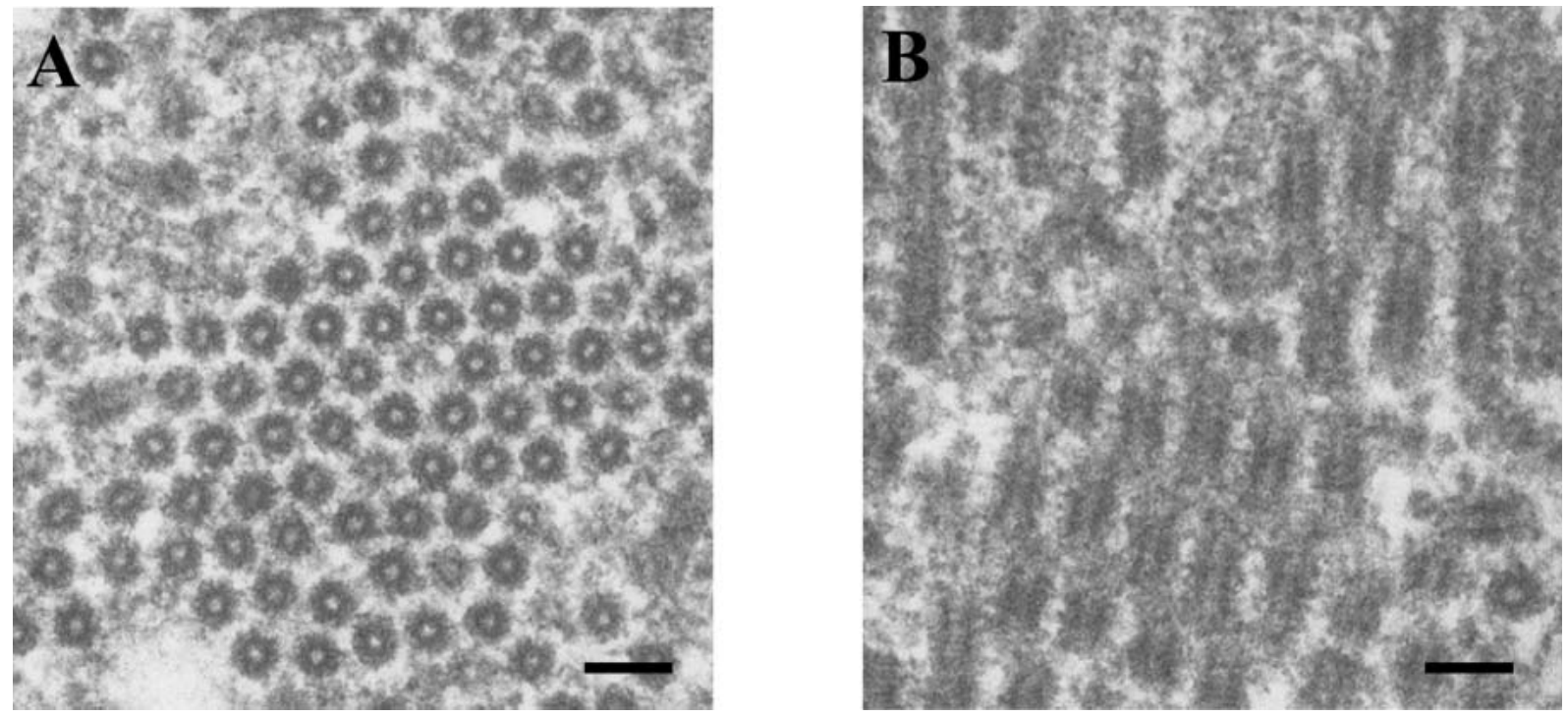

Fig. 1. NC-like structures produced by the expression of NP, VP24, and VP35. Transverse section shows NC-like structures of $50 \mathrm{~nm}$ in diameter with an axial space of $10-15 \mathrm{~nm}$ in the center (A). Longitudinal section of NC-like structures (B). Bars; $100 \mathrm{~nm}$.

Japan Ltd.) and observed in a TECNAI G2 Shera (FEI Company Japan Ltd.) at $200 \mathrm{kV}$. Electron micrographs were taken at 50,000 magnification every $2^{\circ}$ (range from $-70^{\circ}$ to $\left.+70^{\circ}\right)$ and recorded with a CCD camera $(1024 \AA \sim 1024$ pixels with $0.49 \mathrm{~nm} / \mathrm{pixel})$. The alignment of the projections was calculated by R-weighted back projection with IMOD software [11], and these calculations were then used to construct 3D images of the NC-like structures. Reconstructed NC-like structures were visualized with Amira software (Template Graphics Software Inc.). Figure 2 shows galleries of 3D images of NC-like structures at lower and higher magnifications. At the lower magnification, filamentous NC-like structures with an axial channel in the center can be clearly observed (Fig. 2A). Further, ET revealed that these NC-like structures are aligned but are not perfectly parallel, information that has not been obtained by conventional TEM. At the higher magnification (Fig. 2B), the helical grooves spaced at short intervals on the surface of the NClike structures are more visible than those observed in longitudinal sections by conventional TEM (Fig. 1B). In addition, we were able to determine that the helices were lefthanded (Fig. 2B), a finding made only with ET. Thus, ET is a superior tool for acquiring detailed 3D structural information.

The left-handed helix of nucleocapsids has not been reported previously for Ebola virus, but has been documented for other non-segmented negative-strand RNA viruses, such as Sendai virus [5], measles virus [2], simian virus 5, and mumps virus [3]. The nucleocapsids of these viruses also comprise filamentous structures with an axial space in the center, identical to those of Ebola virus. Although the biological significance of this left-handed helix is currently unclear, the structural similarity among these negative-strand RNA viruses suggests common mech- anisms in the morphogenesis of nucleocapsids, incorporation of viral RNAs into nucleocapsids, and transcription and replication of viral RNAs. For this reason, information obtained by further studies on Ebola virus nucleocapsids would likely be applicable to these other viruses.

In conclusion, we have used ET to obtain more detailed structural information on Ebola virus NC-like structures than has been obtained to date using conventional TEM analysis. The ET technique can be applied to complexes as large as an entire virus particle [9], and when combined with cryo-electron microscopy, can provide 3D structures of individual pleiomorphic macromolecules at a near-molecular resolution [12]. Thus, ET is a powerful method to generate fundamental structural information and its application in diverse biological fields should provide a wealth of information regarding the three-dimensional arrangement of cellular components.

ACKNOWLEDGEMENTS. We thank Taiki Watanabe for assistance with the ET experiments and Susan Watson for editing the manuscript. This work was supported by grantsin-aid from the Ministry of Education, Culture, Sports, Science and Technology and the Ministry of Health, Labor and Welfare, Japan, Core Research for Evolutional Science and Technology (CREST) grants from the Japan Science and Technology Agency (JST), Japan, and by grants from the National Institutes of Health, NIAID. TN is the recipient of a Research Fellowship from the Japan Society for the Promotion of Science for Young Scientists.

\section{REFERENCES}

1. Baumeister, W. 2002. Curr. Opin. Struct. Biol. 12: 679-684.

2. Bhella, D., Ralph, A. and Yeo, R.P. 2004. J. Mol. Biol. 340: 
A
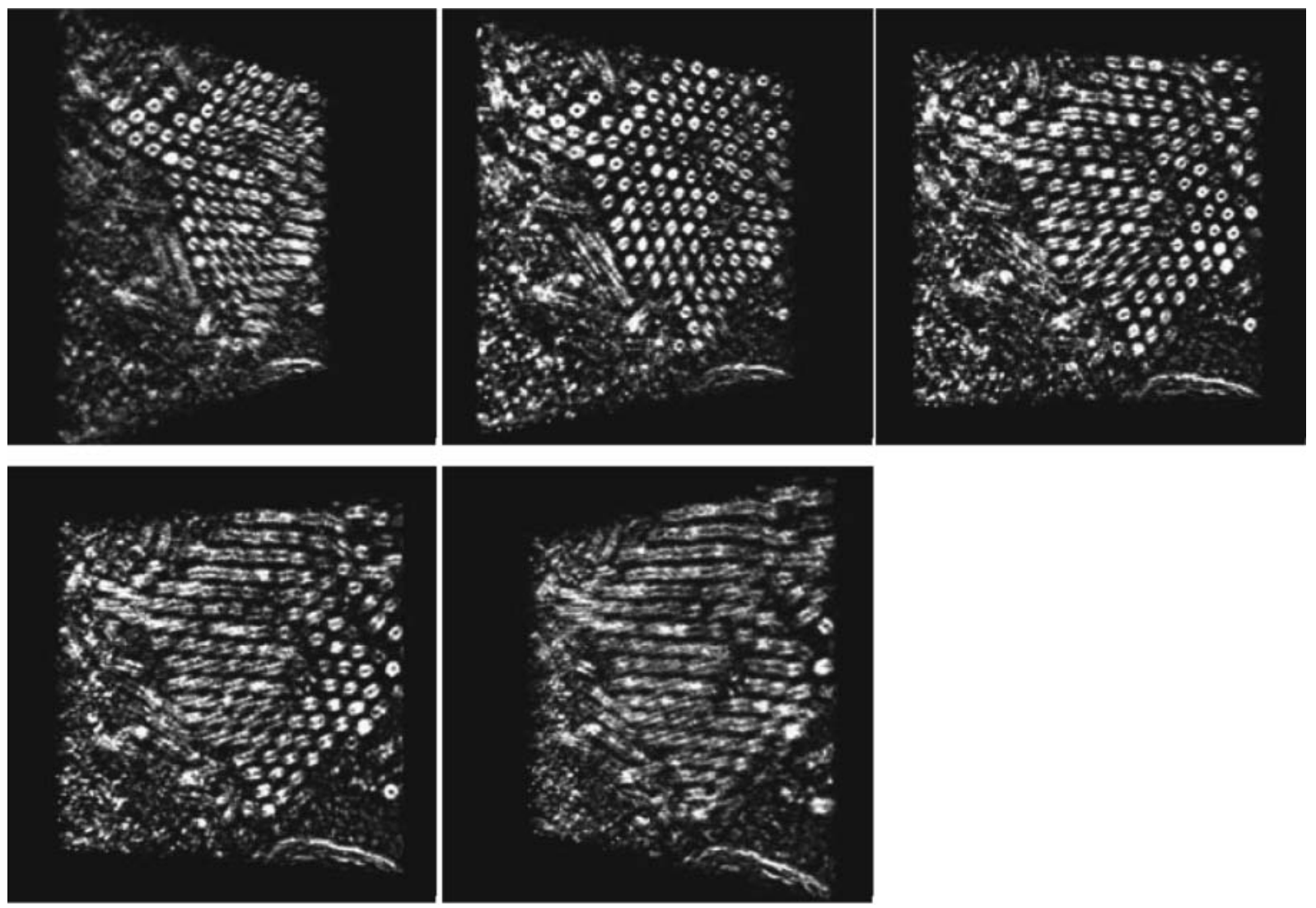

B
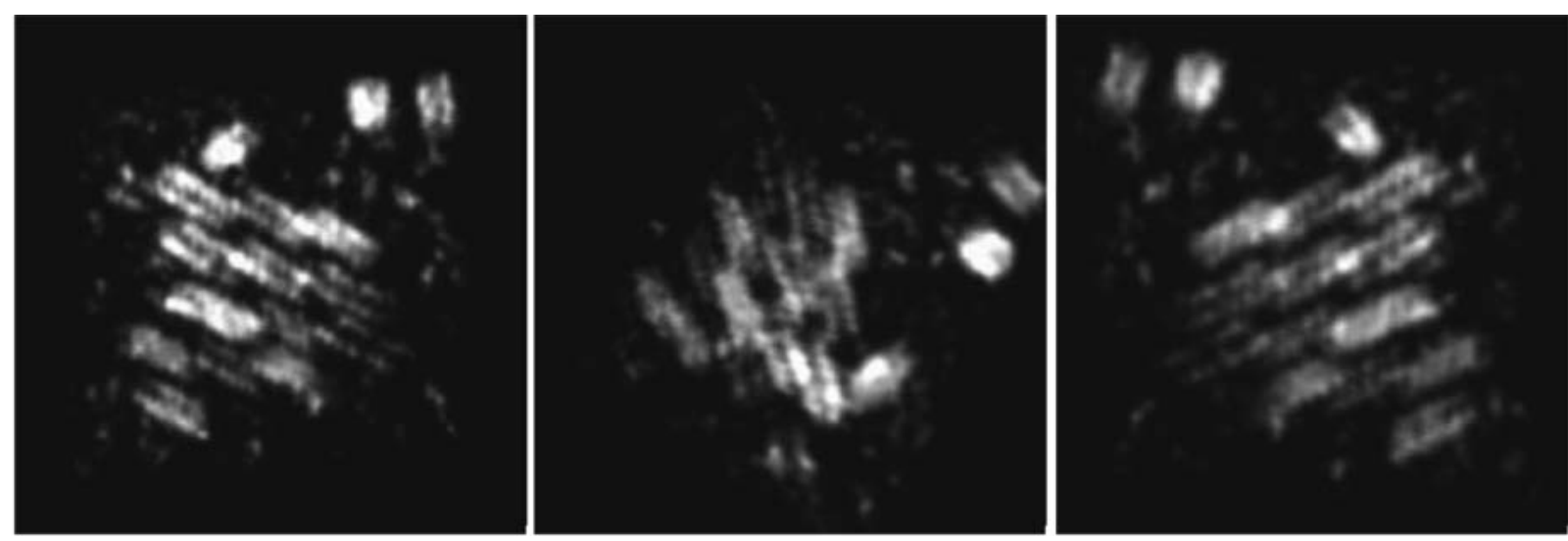

Fig. 2. Reconstructed NC-like structures by ET. Reconstructed NC-like structures were obtained from a 141 tilt series of $200 \mathrm{~nm}$-thick sections, reconstructed by R-weighted back projection with IMOD, and visualized with Amira software. The galleries of 3D NC-like structures are shown at a low (A) and a high (B) magnification.

319-331.

3. Compans, R.W., Mountcatsle, W.E. and Choppin, P.W. 1972. J. Mol. Biol. 65: 167-169.

4. DeRosier, D.J. and Klug, A. 1968. Nature (Lond.) 217: 130134.

5. Egelman, E.H., Wu, S.S., Amrein, M., Portner, A. and Murti,
G. 1989. J. Virol. 63: 2233-2243.

6. Feldmann, H. and Klenk, H.D. 1996. pp. 1-52. In: Advances in virus research, vol. 47 (Maramorosh, K., Murphy, F.A., Shatkin, A.J. eds.), Academia Press, Inc.

7. Frank, J. 1995. Curr. Opin. Struct. Biol. 5: 1194-1201.

8. Geisbert, T.W. and Jahrling, P.B. 1995. Virus. Res. 39: 129- 
150.

9. Grunewald, K., Desai, P., Winkler, D.C., Heymann, J.B., Belnap, D.M., Baumeister, W. and Steven, A.C. 2003. Science 302: 1396-1398.

10. Huang, Y., Xu, L., Sun, Y. and Nabel, G.J. 2002. Mol. Cell. 10: 307-316.

11. Kremer, J.R., Mastronarde, D.N. and McIntosh, J.R. 1996. J. Struct. Biol. 116: 71-76.

12. Medalia, O., Weber, I., Frangakis, A.S., Nicastro, D., Gerisch,
G. and Baumeister, W. 2002. Science 298: 1209-1213.

13. Sanchez, A., Khan, A.S., Zaki, S.R., Nabel, G.J., Ksiazek, T.G. and Peters, C.J. 2001. pp. 1279-1304. In: Fields Virology, 4th ed. (Knipe, D.M., Howley, P.M. eds.), Lippincott/The Williams \& Wilkins Co., Philadelphia, Pa.

14. Watanabe, S., Watanabe, T., Noda, T., Takada, A., Feldmann, H., Jasenosky, L.D. and Kawaoka, Y. 2004. J. Virol. 78: 9991005. 